\title{
Clustering Wireless Sensor and Wireless Ad Hoc Networks using Dominating Tree Concepts
}

\author{
Ishwar Baidari \\ Asst. Professor \\ Dept of Computer Science \\ Karnatak University,Dharwad
}

\author{
H B Walikar, PhD. \\ Professor \\ Dept of Computer Science \\ Karnatak University,Dharwad
}

\author{
Shridevi Shinde \\ Research Scholer \\ Dept of Computer Science \\ Karnatak University,Dharwad
}

\begin{abstract}
The most important criterion for achieving the maximum performance in wirless sensor and ad-hoc networks is to clustering the nodes. Many clustering schemes have been proposed for different ad-hoc networks. In sensor networks the energy stored in the network nodes is limited and usually infeasible to recharge. The clustering schemes for these networks therefore aim at maximizing the energy efficiency. In mobile ad hoc networks the moment of the network nodes may quickly change the topology resulting in the increase of the overhead message in topology maintenance, the clustering schemes for mobile ad hoc networks therefore aim at handling topology maintenance, managing node movement or reducing overhead. In this paper we proposed an algorithm which gives connected dominating set of a tree.
\end{abstract}

\section{Keywords}

Wireless sensor networks, ad-hoc networks, connected dominating set connected dominating tree, tree.

\section{INTRODUCTION}

Many clustering schemes have been proposed [14, 17, 18, 19, $20,21,22]$ for different ad hoc networks and play an important role in self organizing them. A systematic classification of these clustering schemes enables one to better understand and make improvements. This chapter considers the ad hoc sensor network clustering schemes and mobile ad hoc network clustering schemes. In sensor networks, the energy stored in the network nodes is limited and usually infeasible to recharge; the clustering schemes for these networks therefore aim at maximizing the energy efficiency. In mobile ad hoc networks, the movement of the network nodes may quickly change the topology resulting in the increase of the overhead message in topology maintenance; the clustering schemes for mobile ad hoc networks therefore aim at handling topology maintenance, managing node movement or reducing overhead.

Ad hoc network is a self-organizing multihop system of wireless nodes which can communicate with each other without pre-existing infrastructure. A lot of research has been conducted in this field and two types of topology structures have been proposed: flat topology structure and hierarchical clustering structure. The peer to peer flat structure results in a complicated topology in large ad hoc networks and an unstable topology in mobile ad hoc networks because the movement of one node may change the whole topology of the network. Therefore, flat topology structure is inefficient for ad hoc networks. Researchers then proposed hierarchal clustering structures for ad hoc networks. With an ad hoc clustering network, the nodes are separated into groups called clusters. In each cluster, one node is elected as a $\mathrm{CH}$ to act as a local controller. The size of the cluster (the number of nodes in the cluster) depends on the transmission range of the nodes in single hop cluster and the number of hops made by the cluster in multi-hop clusters. The normal node sends or relays data to the $\mathrm{CH}$ which transfers the collected packets to the next hop. The gateway node, belonging to more than one cluster, bridges the $\mathrm{CHs}$ in those clusters. Both $\mathrm{CHs}$ and gateway nodes form the backbone. The following is a list of some advantages of clustering scheme. 1) Only the $\mathrm{CHs}$ and gateway nodes form the backbone network, results in much simpler topology, less overhead, flooding and collision. 2) The change of nodes only affects part topology of the networks, making the topology more stable. 3) Only CHs or gateway nodes need to maintain the route information..

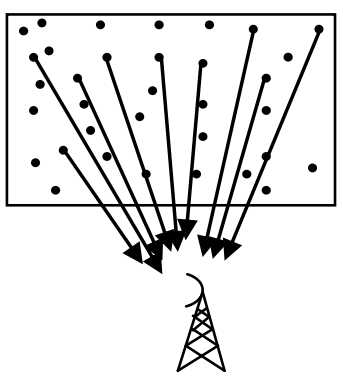

(a)

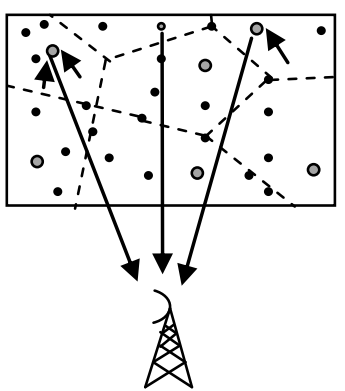

(c)

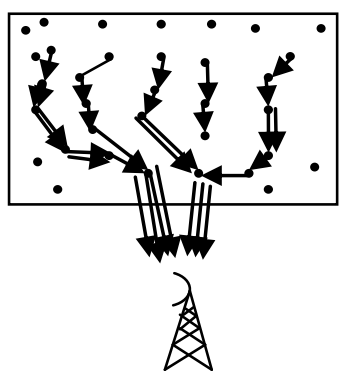

(b)

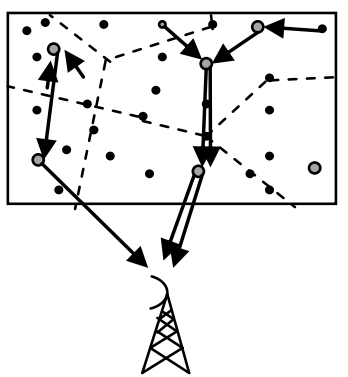

(d)
Figure1. Sensor information forwarding with and without clustering and aggregation. (a) Single hop without clustering.

(b) Multihop without clustering.

\section{RELATED WORK}

Recent years have witnessed an increasing interest in using wireless sensor networks (WSNs) in many applications, including environmental monitoring and military field 
surveillance. In these applications, tiny sensors are deployed and left unattended to continuously report parameters such as temperature, pressure, humidity, light, and chemical activity. Reports transmitted by these sensors are collected by observers (e.g., base stations). The dense deployment and unattended nature of WSNs make it quite difficult to recharge node batteries. Therefore, energy efficiency is a major design goal in these networks. Several WSN applications require only an aggregate value to be reported to the observer. In this case, sensors in different regions of the field can collaborate to aggregate their data and provide more accurate reports about their local regions. For example, in a habitat monitoring application the average reported humidity values may be sufficient for the observer. In military fields where chemical activity or radiation is measured, the maximum value may be required to alert the troops. In addition to improving the fidelity of the reported measurements, data aggregation reduces the communication overhead in the network, leading to significant energy savings. In order to support data aggregation through efficient network organization, nodes can be partitioned into a number of small groups called clusters. Each cluster has a coordinator, referred to as a cluster head, and a number of member nodes. Clustering results in a twotier hierarchy in which cluster heads $(\mathrm{CHs})$ form the higher tier while member nodes form the lower tier. The member nodes report their data to the respective $\mathrm{CHs}$. The $\mathrm{CHs}$ aggregate the data and send them to the central base station through other CHs. Because $\mathrm{CHs}$ often transmit data over longer distances, they lose more energy compared to member nodes. The network may be reclustered periodically in order to select energy-abundant nodes to serve as $\mathrm{CHs}$, thus distributing the load uniformly on all the nodes. Besides achieving energy efficiency, clustering reduces channel contention and packet collisions, resulting in better network throughput under high load. Clustering has been shown to improve network lifetime, a primary metric for evaluating the performance of a sensor network. Although there is no unified definition of "network lifetime," as this concept depends on the objective of an application, common definitions include the time until the first/last node in the network depletes its energy and the time until a node is disconnected from the base station. Even though the objective of several protocols was not to maximize network lifetime, lifetime improvements can still be achieved if data aggregation is exploited and the network is re clustered periodically. Clustering has been extensively studied in the data processing and wired network literatures. The clustering approaches developed in these areas cannot be applied directly to WSNs due to the unique deployment and operational characteristics of these networks. Specifically, WSNs are deployed in an ad hoc manner and have a large number of nodes. The nodes are typically unaware of their locations. Hence, distributed clustering protocols that rely only on neighborhood information are preferred for WSNs (however, most studies in this area still assume that the network topology is known to a centralized controller). Furthermore, nodes in WSNs operate on battery.

\section{Clustering Algorithm}

The main objective of our approach is to cluster sensor network efficiently around few high-energy gateway nodes. The clustering algorithm is responsible for dividing the whole network topology into clusters. The algorithm takes the set of sensor nodes and gateways, and partitions them such that there is one gateway and a subset of the set of nodes in each cluster. Clustering enables[14,17,18,19,20,21,22] network scalability to large number of sensors and extends the life of the network by allowing the sensors to conserve energy through communication with closer nodes and by balancing the load among the gateway nodes. Gateways associate cost to communicate with each sensor in the network. Clusters are formed based on the cost of communication and the load on the gateways. Network setup is performed in two stages; 'Bootstrapping' and 'Clustering'. In the bootstrapping phase, gateways discover the nodes that are located within their communication range. Gateways broadcast a message indicating the start of clustering. We assume that receivers of sensors are open throughout the clustering process. Each gateway starts the clustering at a different instance of time in order to avoid collisions. In reply the sensors also broadcast a message with their maximum transmission power indicating their location and energy reserve in this message. Each node discovered in this phase is included in a range set per gateway. Bootstrapping a sensor network is the processing of establishing inter-node links and forming an overall network topology. Bootstrapping typically consists of two phases:

- Node Discovery: unless the nodes are manually placed, nodes are not aware of their peers and thus should at least discover their neighbors.

- Topology Setup: Based on the established links among neighboring nodes, a network topology should be established to allow for data gathering. In the clustering phase, gateways calculate the cost of communication with each node in the range set. This information is then exchanged between all the gateways. After receiving the data from all the other gateways each gateway start clustering nodes based on the communication cost and the current load on its cluster. When the clustering is over, all the sensors are informed about the ID of the cluster. Clustering Nodes in sensor network they belong to. Since gateways share the common information during clustering, each sensor belongs to only one cluster. For inter-cluster communication all the traffic is routed through the gateways. The clustering algorithm may use a number of metrics to determine how to form clusters. Physical distance of a sensor node from the sensor. In this case, the sensor node determines the nearest gateway and reports to that gateway. Equal number of sensors in each cluster. This ensures that each gateway has equal routing overhead. Redundancy assurance. A sensor may determine that there exists more than one gateway within transmission range. It chooses one gateway and joins that cluster, and keeps the others as backups. Thus, this algorithm may change the cluster formation due to factors such as gateway failure. At first, 
sensor nodes are clustered into clusters and then the CDS algorithm is applied to the clustered network. The algorithm that has been used in our approach uses the proximity-based metrics. Thus, each sensor node chooses the nearest gateway and joins that cluster. All sensors find their Euclidian distance from the gateways and join them. After clustering sensor nodes in clusters with cluster head or gateways, the CDS finding algorithm is used in each cluster. Here we are first deploying the wireless sensor and ad hoc networks in the form of tree and applying the CDS algorithm on tree to retain the connected dominating set in the tree

\section{Proposed Algorithm, for Clustering}

\subsubsection{Tree}

Trees are the simplest connected graphs. These graphs are interesting not only for their theoretical properties but also for their applications.

\subsubsection{Properties of Trees}

A tree as a connected graph without cycles. The following figures are the trees of order 6 .
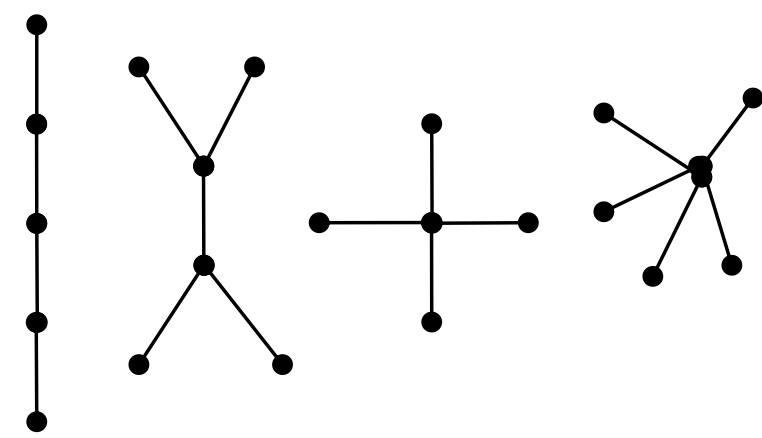

Figure 2

\subsubsection{Spanning Tree}

A spanning tree for is a tree that is a tree that connects all the vertices in

\subsection{Dominating Tree[2]}

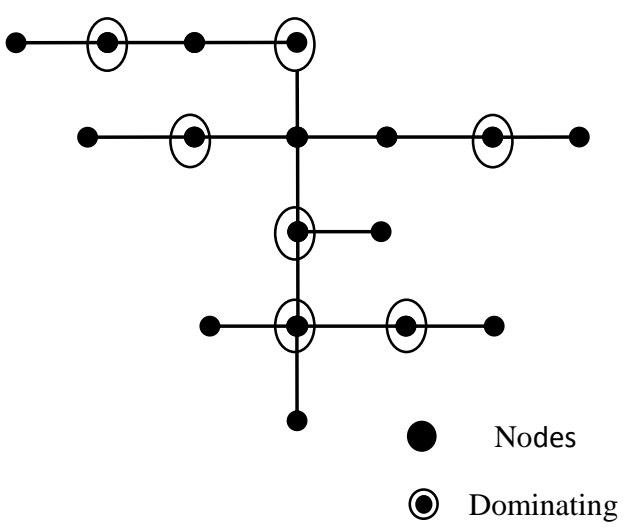

Figure 3

But here our objective is to retain the connected dominating tree which is itself a virtual back bone of the wireless sensor or wireless ad hoc networks. Slightly modifying the liner algorithm for the domination number of a tree designed by $\mathrm{E}$ Cockayne,S Goodman and S Hedetniemi Cock et al [1] ,which gives connected dominating tree. Let us consider the algorithm.

\subsection{Connected Dominating Tree Algorithm} Algorithm DOMSET. To find a D-Set, or MD - Set, DOMSET, in a tree $T$ with free, bound and required vertices.

Step 0. [Initialize] Set DOMSET $\leftarrow \mathcal{O}_{;}^{*} G \leftarrow T$.

Step 1. [Delete $M-1$ end vertices one at a time]

Do

Step 2. G has a free end vertex $\mathrm{v}$ adjacent to a vertex $\mathrm{u}$

Step 3. set $G \leftarrow G-v$.

Step 4. G has a bound end vertex w adjacent to vertex w

Step 5.Relabel as "required"

Step 6. Set $G \leftarrow G-v$.

Step7. G has a required end vertex v adjacent to a vertex 4 Step 8. Set DOMSET $\leftarrow$ DOMSET U $\{$ D?

Step 9.If $\boldsymbol{u}$ is bound then label $u$ as required

Step 10. Set $G \leftarrow G-v$.

od

Step11. [Process last vertex] If the last vertex $\mathrm{w}$ is not free

$$
\text { then DOMSET } \leftarrow \text { DOMSET } \cup\{\mathrm{D}\} \text {. }
$$

Finally after applying above algorithm we get a connected dominating tree as shown in the following figure 4.

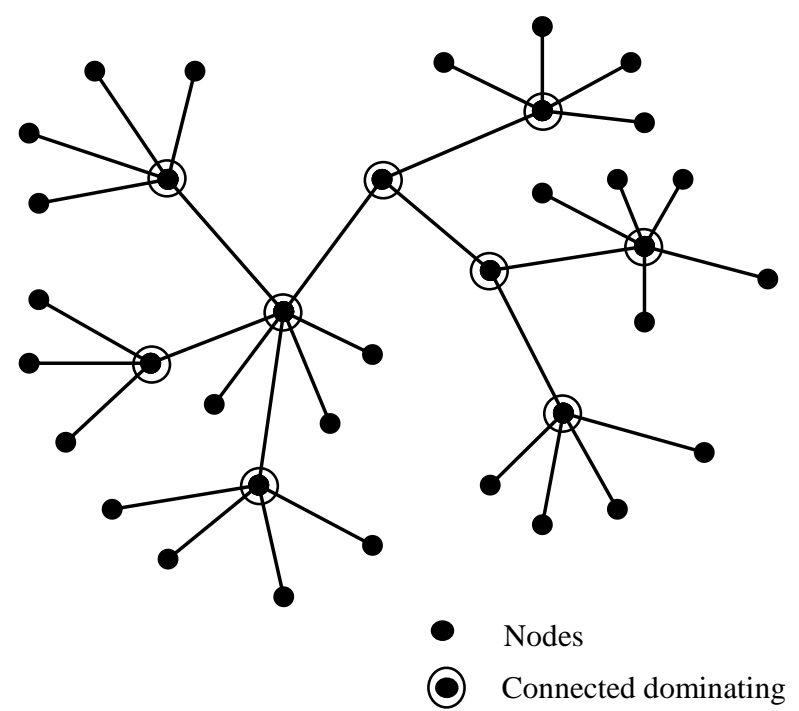

Figure 4 
The following algorithm which computes value of $y$ for an arbitrary unweighted tree . It is taken from Mitchell, E. Cockayne and Hedetniemi [2] and is a linear implementation of the algorithm [1]

\section{Conclusion}

Algorithm presented in this chapter gives clustering based on connected dominating tree concepts .Complexity of the algorithm is linear time. Still we have to consider many challenges of wireless sensor and wireless ad hoc networks, using these algorithms. For example load balancing, energy efficiency and fault tolerance etc.

\section{REFERENCES}

[1] E Cockayne,S. Goodman, and S.Hedetniemi, A Linear Algorithm for the Domination Number of A Tree. Inform.Process.Lett., Volume 4:41-44 number 2,1975

[2] S.L.Mitchell,E.J.Cockayne, and S.T.Hedetniemi.Linear algorithms on recursive representa tion of trees. J.Compute.System Sci.,18(1):76-85,1979.

[3] J. Polastre, J. Hill and D. Culler, Versatile Low Power Media Access for Wireless Sensor Networks,ACM SenSys, November 2004.

[4] T. He, J. Stankovic, C. Lu and T. Abdelzaher, A Spatiotemporal Communication Protocol for Wireless Sensor Networks, IEEE Transactions on Parallel and Distributed Systems, to appear

[5] B. Karp and H. T. Kung, GPSR: Greedy Perimeter Stateless Routing for Wireless Sensor Networks,IEEE Mobicom, August 2000."

[6] C. Intanagonwiwat, R. Govindan, and D. Estrin. Directed diffusion: A scalable and robust communication paradigm for sensor networks. In Proceedings of the 6th Annual International Conference on Mobile Computing and Networking, pages 56-67, ACM Press,2000.

[7] C. Intanagonwiwat, R. Govindan, D. Estrin, J. Heidemann, and F. Silva. Directed diffusion for wireless sensor networking. IEEE/ACM Transactions on Networking,11(1):2-16, 2003.

[8] J. Agre and L. Clare. An integrated architecture for cooperative sensing networks. IEEE Computer, pages 106-108, May 2000..

[9]. West, D. : Introduction to Graph Theory, Second edition, Prentice Hall, Upper Saddle River, N.J., (2001).

[10] Haynes, T., W., Hedetniemi, S., T. and Slater, P., J. : Domination in Graphs,Advanced Topics, Marcel Dekker, Inc., (1998).

[11] Chen, Y., Z., P., Liestman, A., L. and Jiangchuan, L. : Clustering Algorithms for Ad Hoc Wireless Networks, Nova Science Publishers, (2004).

[12] Baker, D. and Ephremides, A. : The Architectural Organization of a Mobile Radio Network via a
Distributed Algorithm, Communications, IEEE Transactions, (1981), 29(11), 1694-1701.

[13] Gerla, M. and Jack T., C., T. : Multicluster, Mobile, Multimedia Radio Network,Wireless Networks, 1(3), (1995), 255-265.

[14] Chen, G., Nocetti, F.,G., Gonzalez and J.S., Stojmenovic, I. : Connectivity Based K-Hop Clustering in Wireless Networks, System Sciences, Proc. of the 35th Annual Hawaii International Conference, (2002), 2450-2459.

[15] Ohta, T., Inoue, S. and Kakuda, Y. : An Adaptive Multihop Clustering Scheme for Highly Mobile Ad Hoc Networks, Proc. of 6th ISADS'03, (2003).

[16] Chen, Y., P. and Liestman, A., L. : A Zonal Algorithm for Clustering Ad Hoc Networks, International Journal of Foundations of Computer Science, (2003), 14(2),305322.

[17] Stojmenovic, I., Seddigh M. and Zunic, J. : Dominating Sets and Neighbor Elimination-Based Broadcasting Algorithms in Wireless Networks, IEEE Transactions on Parallel and Distributed Systems, (2002), 13, 14-25."

[18] Guha S. and Khuller, S. : Approximation Algorithms for Connected Dominating Sets, Springer Verlag New York, LLC, ISSN: 0178-4617, (1998).

[19] Das, B. and Bharghavan, V. : Routing in Ad-Hoc Networks Using Minimum Connected Dominating Sets, Communications, ICC97 Montreal, 'Towards the Knowledge Millennium', IEEE International Conference, (1997), 1, 376-380

[20] Wu and Li [14]," Wu, J. and Li, H. : A Dominating-SetBased Routing Scheme in Ad Hoc Wireless Networks, Springer Science Business Media B.V., Formerly Kluwer Academic Publishers B.V. ISSN: 1018-4864, (2001).

[21] Wu, J. and Li, H. : A Dominating-Set-Based Routing Scheme in Ad Hoc Wireless Networks, Springer Science+Business Media B.V., Formerly Kluwer Academic Publishers B.V. ISSN: 1018-4864, (2001).

[22] Wan, P., J., Alzoubi, K., M. and Frieder, O. : Distributed Construction of Connected Dominating Set in Wireless Ad Hoc Networks, Springer Science+Business Media B.V., Formerly Kluwer Academic Publishers B.V., , (2002), 9(2), 141-149

[23] "Liu, H., Pan, Y. and Cao, J. : An Improved Distributed Algorithm for Connected Dominating Sets in Wireless Ad Hoc Networks, Parallel and Distributed Processing and Applications, Proc. of the ISPA 2004, (2004), 340.

[24] Liu, H., Pan, Y. and Cao, J. : An Improved Distributed Algorithm for Connected Dominating Sets in Wireless Ad Hoc Networks, Parallel and Distributed Processing and Applications, Proc. of the ISPA 2004, (2004), 340.

[25] J.Liu, Maximal Independent Sets in Bipartite Graphs, Journal of Graph Theory 17(1993)495-507. 